\title{
Stability Analysis for Impulsive Stochastic Reaction-Diffusion Differential System and Its Application to Neural Networks
}

\author{
Yanke $\mathrm{Du},{ }^{1}$ Yanlu $\mathrm{Li}^{2}{ }^{2}$ and Rui $\mathrm{Xu}^{1}$ \\ ${ }^{1}$ Institute of Applied Mathematics, Shijiazhuang Mechanical Engineering College, Shijiazhuang 050003, China \\ ${ }^{2}$ Training Department, Shijiazhuang Mechanical Engineering College, Shijiazhuang 050003, China
}

Correspondence should be addressed to Yanke Du; yankedu2011@163.com

Received 26 March 2013; Revised 28 June 2013; Accepted 12 July 2013

Academic Editor: Debasish Roy

Copyright (c) 2013 Yanke Du et al. This is an open access article distributed under the Creative Commons Attribution License, which permits unrestricted use, distribution, and reproduction in any medium, provided the original work is properly cited.

\begin{abstract}
This paper is concerned with the stability of impulsive stochastic reaction-diffusion differential systems with mixed time delays. First, an equivalent relation between the solution of a stochastic reaction-diffusion differential system with time delays and impulsive effects and that of corresponding system without impulses is established. Then, some stability criteria for the stochastic reaction-diffusion differential system with time delays and impulsive effects are derived. Finally, the stability criteria are applied to impulsive stochastic reaction-diffusion Cohen-Grossberg neural networks with mixed time delays, and sufficient conditions are obtained for the exponential $p$-stability of the zero solution to the neural networks. An example is given to illustrate the effectiveness of our theoretical results. The systems we studied in this paper are more general, and some existing results are improved and extended.
\end{abstract}

\section{Introduction}

In recent years, impulsive dynamical systems have attracted considerable attention due to its wide applications in the areas of economics, physics, population dynamics, engineering, biology, and so on. These systems arise because they are subject to abrupt state changes at certain moments of time, and these changes may be related to such phenomena as shocks, harvesting, or other faults. Meanwhile, time delays are frequently encountered in real world, which can cause instability and oscillations in a system. A large number of stability criteria of impulsive delay systems have been reported (see [1-6] and references therein).

Stochastic effects are common phenomena due to disturbances or uncertainties in a system. A lot of dynamical systems have variable structures subject to stochastic abrupt changes, which may result from abrupt phenomena such as stochastic failures and repairs of the components, changes in the interconnections of subsystems, and sudden environment switching. Hence, considerable attention has been paid to the study of stochastic systems, and various interesting results have been reported in the literatures; for example, see [2-4, 7, $8]$ and references therein. In particular, Li et al. [8] considered the following impulsive stochastic differential system with time delay:

$$
\begin{array}{r}
\mathrm{d} y_{i}(t, x)=F_{i}\left(t, y_{1}(t), \ldots, y_{n}(t), y_{1}(t-\tau(t)),\right. \\
\left.\ldots, y_{n}(t-\tau(t))\right) \mathrm{d} t \\
+G_{i}\left(t, y_{1}(t), \ldots, y_{n}(t), y_{1}(t-\tau(t)),\right. \\
\left.\ldots, y_{n}(t-\tau(t))\right) \mathrm{d} w_{i}(t), \quad t \neq t_{k}, \\
y_{i}\left(t_{k}^{+}\right)-y_{i}\left(t_{k}\right)=I_{k i}\left(y_{1}\left(t_{k}\right), \ldots, y_{n}\left(t_{k}\right)\right), \\
t=t_{k}, \quad k \in N, \quad i=1,2, \ldots, n .
\end{array}
$$

They showed the stability results of system (1) by transforming (1) into an equivalent system without impulses.

Generally speaking, diffusion effects cannot be avoided in systems modeling many real world phenomena. As a representation example in neural networks, when electrons are moving in an asymmetric electromagnetic field, it inevitably leads to diffusion phenomena. In [5, 9-11], the stabilities of the equilibrium points of some types of neural networks 
with reaction-diffusion terms have been investigated. On the other hand, distributed delay systems can characterize the cumulative effects of the past values of the dynamic and are often used to model the time lag phenomena in thermodynamics, ecology, epidemiology, and neural networks. For example, neural networks usually have a spatial extent due to the presence of a multitude of parallel pathways with a variety of axon sizes and lengths. It is desired to model them by introducing continuously distributed delays over a certain duration of time such that the distant past has less influence compared to the recent behavior of the state.

However, in [8], the authors neglected the effects of diffusion and distributed delays. To the best of our knowledge, there are few results about the stability of impulsive stochastic reaction-diffusion deferential systems (ISRDDSs) with mixed time delays. Motivated by [8] and the previous discussions, we are concerned with the stability of the following ISRDDS with time-varying discrete delays and distributed delays:

$$
\begin{aligned}
& \mathrm{d} u(t, x)= \nabla(D(t, x, u(t, x)) \circ \nabla u(t, x)) \mathrm{d} t \\
&+F\left(t, u(t, x), u\left(t-\tau_{1}(t), x\right), \ldots,\right. \\
&\left.u\left(t-\tau_{p}(t), x\right), \int_{t-r(t)}^{t} u(s, x) \mathrm{d} s\right) \mathrm{d} t \\
&+G\left(t, u(t, x), u\left(t-\tau_{1}(t), x\right), \ldots,\right. \\
& u\left(t-\tau_{p}(t), x\right), \\
&\left.\int_{t-r(t)}^{t} u(s, x) \mathrm{d} s\right) \mathrm{d} w(t), \quad t \neq t_{k}, \\
& u\left(t_{k}^{+}, x\right)- u\left(t_{k}, x\right)=I_{k}\left(u\left(t_{k}, x\right)\right), \\
& u(t, x)=\phi(t, x), \quad t=t_{k}, \quad k \in N, \\
&(t, x) \in[-\gamma, 0] \times \Omega, \quad \Omega \in \mathbb{R}^{m},
\end{aligned}
$$

in which

$$
\begin{gathered}
u(t, x)=\left(u_{1}(t, x), u_{2}(t, x), \ldots, u_{n}(t, x)\right)^{\mathrm{T}}, \\
u\left(t-\tau_{j}(t), x\right)=\left(u_{1}\left(t-\tau_{j}(t), x\right),\right. \\
u_{2}\left(t-\tau_{j}(t), x\right), \ldots, \\
\left.u_{n}\left(t-\tau_{j}(t), x\right)\right)^{\mathrm{T}}(j=1,2, \ldots, p), \\
\int_{t-r(t)}^{t} u(s, x) \mathrm{d} s=\left(\int_{t-r_{1}(t)}^{t} u_{1}(s, x) \mathrm{d} s, \int_{t-r_{2}(t)}^{t} u_{2}(s, x) \mathrm{d} s,\right. \\
\left.\ldots, \int_{t-r_{n}(t)}^{t} u_{n}(s, x) \mathrm{d} s\right)^{\mathrm{T}}, \\
F=\left(F_{1}, \ldots, F_{n}\right)^{\mathrm{T}}, \quad G=\left(G_{1}, \ldots, G_{n}\right)^{\mathrm{T}},
\end{gathered}
$$

$$
\begin{gathered}
I_{k}=\left(I_{k 1}, \ldots, I_{k n}\right)^{\mathrm{T}} \quad(k \in N), \\
\phi=\left(\phi_{1}, \ldots, \phi_{n}\right)^{\mathrm{T}}, \\
D(t, x, u(t, x))=\left(D_{i l}(t, x, u(t, x))\right)_{n \times m}, \\
\nabla u=\left(\nabla u_{1}, \ldots, \nabla u_{n}\right)^{\mathrm{T}}, \quad \nabla u_{i}=\left(\frac{\partial u_{i}}{\partial x_{1}}, \ldots, \frac{\partial u_{i}}{\partial x_{m}}\right), \\
D \circ \nabla u=\left(D_{i l}(t, x, u(t, x)) \frac{\partial u_{i}}{\partial x_{l}}\right)_{n \times m}, \\
Y=\left(Y_{1}, \ldots, Y_{n}\right)^{\mathrm{T}}, \quad Y_{i}=\left(y_{i 1}, \ldots, y_{i m}\right)^{\mathrm{T}}, \\
\nabla \cdot Y_{i}=\sum_{l=1}^{m} \frac{\partial y_{i l}}{\partial x_{l}}, \quad \nabla \cdot Y=\left(\nabla \cdot Y_{1}, \ldots, \nabla \cdot Y_{n}\right)^{\mathrm{T}} .
\end{gathered}
$$

$u_{i}(t, x)$ is the state variable, $x_{i}$ is the space variable, $D_{i l}(t, x, u(t, x)) \geq 0$ is a diffusion operator, $\tau_{j}(t)$ and $r_{i}(t)$ are time-varying functions, $w(t)=\left(w_{1}(t), w_{2}(t), \ldots, w_{n}(t)\right)^{\mathrm{T}} \in$ $\mathbb{R}^{n}$ is a Brownian motion defined on a complete probability space $(S, \mathscr{F}, \mathscr{P})$, and $I_{k i}$ is the impulsive function; $\gamma=$ $\max _{1 \leq j \leq p, 1 \leq i \leq n}\left\{\tau_{j}, r_{i}\right\}, \tau_{j}=\sup _{t \geq 0} \tau_{j}(t)$, and $r_{i}=\sup _{t \geq 0} r_{i}(t)$.

The organization of this paper is as follows. In Section 2, some preliminaries are given. In Section 3, by transforming the solutions of the stochastic reaction-diffusion differential system with delay and impulsive effects into that of the corresponding system without impulses, some stability criteria for the stochastic reaction-diffusion differential system with delay and impulsive effects are derived. In Section 4, the stability criteria are applied to impulsive stochastic reaction-diffusion Cohen-Grossberg neural networks (ISRDCGNNs) with mixed time delays, and sufficient conditions are obtained for the exponential $p$-stability of the zero solution to the neural networks. In Section 5, a numerical example is provided to illustrate the effectiveness of the theoretical results. A concluding remark is given in Section 6 to end this work.

\section{Preliminaries}

For convenience, we introduce several notations. Let $P C\left([-\gamma, 0] \times \Omega, \mathbb{R}^{n}\right):=\left\{u(t, x):[-\gamma, 0] \times \Omega \rightarrow \mathbb{R}^{n} \mid u(t, x)\right.$ is continuous at $t \neq t_{k}, u\left(t_{k}^{-}, x\right)=u\left(t_{k}, x\right)$, and $u\left(t_{k}^{+}, x\right)$ exists for $\left.t_{k}\right\}$. For $u(t, x)=\left(u_{1}(t, x), \ldots, u_{n}(t, x)\right)^{\mathrm{T}} \in \mathbb{R}^{n}$, we define

$$
\|u(t, x)\|=\sum_{i=1}^{n}\left\|u_{i}(t, x)\right\|=\sum_{i=1}^{n}\left(\int_{\Omega}\left|u_{i}(t, x)\right|^{p} \mathrm{~d} x\right)^{1 / p}
$$

and for $\phi(s, x)=\left(\phi_{1}(s, x), \ldots, \phi_{n}(s, x)\right)^{\mathrm{T}} \in P C([-\gamma, 0] \times$ $\left.\Omega, \mathbb{R}^{n}\right)$, we define $\|\phi(s, x)\|=\sup _{-\gamma \leq s \leq 0} \sum_{i=1}^{n}\left\|\phi_{i}(s, x)\right\|$. Denote $P C_{F_{0}}^{b}\left([-\gamma, 0] \times \Omega, \mathbb{R}^{n}\right):=\{u(t, x) \in P C([-\gamma, 0] \times$ $\left.\Omega, \mathbb{R}^{n}\right) \mid u(t, x)$ is bounded, $F_{0}$-measurable, and $E\|u(t, x)\|<$ $\infty\}, P C_{F_{0}}^{b}(\delta):=\left\{u(t, x) \in P C_{F_{0}}^{b}\left([-\gamma, 0] \times \Omega, \mathbb{R}^{n}\right) \mid E\|u(t, x)\| \leq\right.$ $\delta\}$. Throughout this paper, we always assume that a product equals to unity if the number of factors is zero. 
Definition 1. A function $u(t, x):[-\gamma, 0] \times \Omega \rightarrow \mathbb{R}^{n}$ is said to be the solution of system (2) if the following conditions are satisfied:

(i) $u(t, x)$ is piecewise continuous with the first kind discontinuity at the points $t_{k}, k \in N$. Moreover, $u(t, x)$ is left continuous at each point,

(ii) $u(t, x)$ satisfies system (2).

Definition 2. The zero solution of system (2) is said to be as follows.

(i) $p$-stable if, for any $\varepsilon>0$, there exists a $\delta>0$ such that the initial function $\phi \in P C_{F_{0}}^{b}(\delta)$ implies $E\|u(t, x)\|^{p}<$ $\varepsilon$ for $(t, x) \in(0,+\infty) \times \Omega$. Especially, when $p=1$, it is said to be stable.

(ii) Exponentially $p$-stable if there is a pair of positive constants $\lambda$ and $K$ such that, for any initial condition $\phi \in P C_{F_{0}}^{b}\left([-\gamma, 0] \times \Omega, \mathbb{R}^{n}\right)$, there holds $E\|u(t, x)\|^{p} \leq$ $K\|\phi\|^{p} e^{-\lambda t}, t \geq 0$. Here, $\lambda$ is called the exponential convergence rate. When $p=2$ especially, it is said to be exponentially stable in mean square.

(iii) Asymptotically stable if it is stable, and there exists a $\delta>0$ such that the initial function $\phi \in P C_{F_{0}}^{b}(\delta)$ implies $\lim _{t \rightarrow \infty} E\|u(t, x)\|=0$.

For system (2), one makes the following assumptions:

(H1) $0 \leq t_{0}<t_{1}<t_{2}<\cdots<t_{k}<\cdots$ are fixed impulsive moments such that $t_{k} \rightarrow \infty$ as $k \rightarrow \infty$;

(H2) $F, G: R_{+} \times \underbrace{R^{n} \times \cdots \times R^{n}}_{p+2} \rightarrow R^{n}$ satisfy $F(t, 0, \ldots, 0) \equiv$ 0 and $G(t, 0, \ldots, 0) \equiv 0$;

(H3) $I_{k}: R^{n} \rightarrow R^{n}$ satisfies $I_{k}(0)=0, k \in N$;

(H4) except for the zero solution of (2), for any solution $u(t, x)$ of $(2), u(t, x) \neq 0$ and $I_{k}\left(u\left(t_{k}, x\right)\right) \neq-u\left(t_{k}, x\right)$, $k \in N$.

Denoting

$$
\begin{array}{r}
J_{k i}:=\frac{u_{i}\left(t_{k}, x\right)}{u_{i}\left(t_{k}, x\right)+I_{k i}\left(u_{1}\left(t_{k}, x\right), \ldots, u_{n}\left(t_{k}, x\right)\right)}, \\
i=1,2, \ldots, n, \quad k \in N,
\end{array}
$$

we consider the following delayed stochastic reactiondiffusion differential system without impulses:

$$
\begin{gathered}
\mathrm{d} v(t, x)=\nabla \cdot(\widetilde{D}(t, x, v(t, x)) \circ \nabla v(t, x)) \mathrm{d} t \\
+\prod_{0 \leq t_{k}<t} J_{k} \widetilde{F}\left(t, v(t, x), v\left(t-\tau_{1}(t), x\right), \ldots,\right. \\
v\left(t-\tau_{p}(t), x\right), \\
\left.\quad \int_{t-r(t)}^{t} v(s, x) d s\right) \mathrm{d} t
\end{gathered}
$$

$$
\begin{gathered}
+\prod_{0 \leq t_{k}<t} J_{k} \widetilde{G}\left(t, v(t, x), v\left(t-\tau_{1}(t), x\right), \ldots,\right. \\
v\left(t-\tau_{p}(t), x\right), \\
\left.\quad \int_{t-r(t)}^{t} v(s, x) \mathrm{d} s\right) \mathrm{d} w(t), \\
v(t, x)=\phi(t, x), \quad(t, x) \in[-\gamma, 0] \times \Omega,
\end{gathered}
$$

where

$$
\begin{aligned}
& J_{k}=\operatorname{diag}\left(J_{k 1}, J_{k 2}, \ldots, J_{k n}\right) \text {, } \\
& \widetilde{D}(t, x, v(t, x))=D\left(t, x, \prod_{0 \leq t_{k}<t} J_{k}^{-1} v(t, x)\right), \\
& \widetilde{F}\left(t, v(t, x), v\left(t-\tau_{1}(t), x\right), \ldots,\right. \\
& \left.v\left(t-\tau_{p}(t), x\right), \int_{t-r(t)}^{t} v(s, x) \mathrm{d} s\right) \\
& =F\left(t, \prod_{0 \leq t_{k}<t} J_{k}^{-1} v(t, x)\right. \text {, } \\
& \prod_{0 \leq t_{k}<t-\tau_{1}(t)} J_{k}^{-1} v\left(t-\tau_{1}(t), x\right), \ldots, \\
& \prod_{0 \leq t_{k}<t-\tau_{p}(t)} J_{k}^{-1} v\left(t-\tau_{p}(t), x\right), \\
& \left.\int_{t-r(t)}^{t} \prod_{0 \leq t_{k}<s} J_{k}^{-1} v(s, x) \mathrm{d} s\right) \\
& \widetilde{G}\left(t, v(t, x), v\left(t-\tau_{1}(t), x\right), \ldots,\right. \\
& \left.v\left(t-\tau_{p}(t), x\right), \int_{t-r(t)}^{t} v(s, x) \mathrm{d} s\right) \\
& =G\left(t, \prod_{0 \leq t_{k}<t} J_{k}^{-1} v(t, x)\right. \text {, } \\
& \prod_{0 \leq t_{k}<t-\tau_{1}(t)} J_{k}^{-1} v\left(t-\tau_{1}(t), x\right), \ldots, \\
& \prod_{0 \leq t_{k}<t-\tau_{p}(t)} J_{k}^{-1} v\left(t-\tau_{p}(t), x\right), \\
& \left.\int_{t-r(t)}^{t} \prod_{0 \leq t_{k}<s} J_{k}^{-1} v(s, x) \mathrm{d} s\right) .
\end{aligned}
$$

A function vector $v(t, x)$ is a solution of $(6)$ on $[-\gamma,+\infty) \times$ $\Omega$ if it is absolutely continuous on $[-\gamma,+\infty) \times \Omega$ and satisfies (6) almost everywhere for $t \geq 0$ and $x \in \Omega$. 


\section{Stability Criteria}

In this section, we first establish an equivalent relation between the solution of system (2) and that of system (6).

Lemma 3. Assume that (H1)-(H4) hold. Then $u(t, x)$ is a solution of system (2) if and only if $v(t, x)$ is a solution of system (6), where $u(t, x)=\prod_{0 \leq t_{k}<t} J_{k}^{-1} v(t, x)$ or $v(t, x)=$ $\prod_{0 \leq t_{k}<t} J_{k} u(t, x)$.

Proof. First, we prove the sufficiency. Letting $v(t, x)$ be a solution of system (6), we derive that, for any $t \neq t_{k}$,

$$
\begin{aligned}
& \mathrm{d} u(t, x)=\mathrm{d}\left(\prod_{0 \leq t_{k}<t} J_{k}^{-1} v(t, x)\right)=\prod_{0 \leq t_{k}<t} J_{k}^{-1} \mathrm{~d}(v(t, x)) \\
& =\prod_{0 \leq t_{k}<t} J_{k}^{-1} \nabla \cdot(\widetilde{D}(t, x, v(t, x)) \circ \nabla v(t, x)) \mathrm{d} t \\
& +\widetilde{F}\left(t, v(t, x), v\left(t-\tau_{1}(t), x\right), \ldots,\right. \\
& \left.v\left(t-\tau_{p}(t), x\right), \int_{t-r(t)}^{t} v(s, x) \mathrm{d} s\right) \mathrm{d} t \\
& +\widetilde{G}\left(t, v(t, x), v\left(t-\tau_{1}(t), x\right),\right. \\
& \ldots, v\left(t-\tau_{p}(t), x\right), \\
& \left.\int_{t-r(t)}^{t} v(s, x) \mathrm{d} s\right) \mathrm{d} w(t) \\
& =\prod_{0 \leq t_{k}<t} J_{k}^{-1} \nabla \cdot\left(D\left(t, x, \prod_{0 \leq t_{k}<t} J_{k}^{-1} v(t, x)\right)\right. \\
& \left.\circ \nabla\left(\prod_{0 \leq t_{k}<t} J_{k} u(t, x)\right)\right) \mathrm{d} t \\
& +F\left(t, \prod_{0 \leq t_{k}<t} J_{k}^{-1} v(t, x)\right. \text {, } \\
& \prod_{0 \leq t_{k}<t-\tau_{1}(t)} J_{k}^{-1} v\left(t-\tau_{1}(t), x\right), \ldots, \\
& \prod_{0 \leq t_{k}<t-\tau_{p}(t)} J_{k}^{-1} v\left(t-\tau_{p}(t), x\right), \\
& \left.\int_{t-r(t)}^{t} \prod_{0 \leq t_{k}<s} J_{k}^{-1} v(s, x) \mathrm{d} s\right) \mathrm{d} t \\
& +G\left(t, \prod_{0 \leq t_{k}<t} J_{k}^{-1} v(t, x),\right. \\
& \prod_{0 \leq t_{k}<t-\tau_{1}(t)} J_{k}^{-1} v\left(t-\tau_{1}(t), x\right), \ldots, \\
& \prod_{0 \leq t_{k}<t-\tau_{p}(t)} J_{k}^{-1} v\left(t-\tau_{p}(t), x\right), \\
& \left.\int_{t-r(t)}^{t} \prod_{0 \leq t_{k}<s} J_{k}^{-1} v(s, x) \mathrm{d} s\right) \mathrm{d} w(t)
\end{aligned}
$$

$$
\begin{gathered}
=\nabla \cdot(D(t, x, u(t, x)) \circ \nabla u(t, x)) \mathrm{d} t \\
+F\left(t, u(t, x), u\left(t-\tau_{1}(t), x\right), \ldots,\right. \\
\left.u\left(t-\tau_{p}(t), x\right), \int_{t-r(t)}^{t} u(s, x) \mathrm{d} s\right) \mathrm{d} t \\
+G\left(t, u(t, x), u\left(t-\tau_{1}(t), x\right), \ldots,\right. \\
u\left(t-\tau_{p}(t), x\right), \\
\left.\int_{t-r(t)}^{t} u(s, x) \mathrm{d} s\right) \mathrm{d} w(t) .
\end{gathered}
$$

On the other hand, for any $k \in N$, we have

$$
\begin{aligned}
u\left(t_{k}^{+}, x\right) & =\lim _{t \rightarrow t_{k}^{+}} \prod_{0 \leq t_{j}<t} J_{j}^{-1} v(t, x)=\prod_{0 \leq t_{j} \leq t_{k}} J_{j}^{-1} v\left(t_{k}^{+}, x\right) \\
& =J_{k}^{-1} \prod_{0 \leq t_{j}<t_{k}} J_{j}^{-1} v\left(t_{k}, x\right)=J_{k}^{-1} u\left(t_{k}, x\right) \\
& =u\left(t_{k}, x\right)+I_{k}\left(u\left(t_{k}, x\right)\right) \\
u\left(t_{k}^{-}, x\right) & =\lim _{t \rightarrow t_{k}^{-}} \prod_{0 \leq t_{j}<t} J_{j}^{-1} v(t, x) \\
& =\prod_{0 \leq t_{j}<t_{k}} J_{j}^{-1} v\left(t_{k}^{-}, x\right) \\
& =\prod_{0 \leq t_{j}<t_{k}} J_{j}^{-1} v\left(t_{k}, x\right)=u\left(t_{k}, x\right) .
\end{aligned}
$$

Further, if $v(t, x)$ is a solution of system (6) with initial condition $v(t, x)=\phi(t, x),(t, x) \in[-\gamma, 0] \times \Omega$, then $u(t, x)=\prod_{0 \leq t_{k}<t} J_{k}^{-1} v(t, x)=v(t, x)=\phi(t, x),(t, x) \epsilon$ $[-\gamma, 0] \times \Omega$. Therefore, $u(t, x)$ is a solution of system (2) with initial condition $u(t, x)=\phi(t, x),(t, x) \in[-\gamma, 0] \times \Omega$, and the sufficiency is proved. Similarly, we can prove the necessity.

Remark 4. Lemma 3 gives the equivalent relation between the solution of a stochastic reaction-diffusion differential delay system with impulsive effects and the solution of a corresponding system without impulses. Based on the "equivalent method," the existence and uniqueness of the solution of a stochastic reaction-diffusion differential delay system with impulsive effects can be derived by a new way; that is, any conditions that ensure the existence and uniqueness of the solution of system (6) without impulses will also ensure the existence and uniqueness of the system (2) with impulses.

In what follows, we will reduce the stabilities of system (2) to those of corresponding system (6). 
Theorem 5. Under assumptions (H1)-(H4), if there exists a constant $M>0$ such that, for any $t>0$,

$$
\left|\prod_{0 \leq t_{k}<t} J_{k i}^{-1}\right| \leq M, \quad i=1,2, \ldots, n,
$$

and the zero solution of (6) is p-stable (exponentially p-stable, asymptotically stable), then the zero solution of (2) is also $p$ stable (exponentially p-stable, asymptotically stable).

Proof. Let $u(t, x)$ and $v(t, x)$ be the solutions of systems (2) and (6), respectively. Since the zero solution of (6) is $p$-stable, we have that, for any $\varepsilon>0$, there exists a scalar $\delta>0$ such that the initial condition $\phi \in P C_{F_{0}}^{b}(\delta)$ implies $E\|v(t, x)\|^{p}<$ $\varepsilon / M^{p}$ for $(t, x) \in(0,+\infty) \times \Omega$. By Lemma $3, u(t, x)=$ $\prod_{0 \leq t_{k}<t} J_{k}^{-1} v(t, x)$ is a solution of $(2)$ on $(t, x) \in[-\gamma,+\infty) \times \Omega$. Furthermore, it is easy to see that

$$
\begin{aligned}
E\|u(t, x)\|^{p} & =E\left\|\left(u_{1}(t, x), \ldots, u_{n}(t, x)\right)^{\mathrm{T}}\right\|^{p} \\
& =E \|\left(\prod_{0 \leq t_{k}<t} J_{k 1}^{-1} v_{1}(t, x), \ldots,\right. \\
& \left.\prod_{0 \leq t_{k}<t} J_{k n}^{-1} v_{n}(t, x)\right)^{\mathrm{T}} \|^{p} \\
& =E\left[\sum_{i=1}^{n}\left(\int_{\Omega}\left|\prod_{0 \leq t_{k}<t} J_{k i}^{-1} v_{i}(t, x)\right|^{p} \mathrm{~d} x\right)^{1 / p}\right]^{p} \\
& \leq E\left[M \sum_{i=1}^{n}\left(\int_{\Omega}\left|v_{i}(t, x)\right|^{p} \mathrm{~d} x\right)^{1 / p}\right]^{p} \\
& =M^{p} E\|v(t, x)\|^{p}<M^{p} \cdot \frac{\varepsilon}{M^{p}}=\varepsilon .
\end{aligned}
$$

Hence, the zero solution of (2) is $p$-stable. Using similar arguments, we can verify that if the zero solution of (6) is exponentially $p$-stable (asymptotically stable), then the zero solution of (2) is also exponentially $p$-stable (asymptotically stable). This completes the proof.

In a similar way, we can derive the following results.

Theorem 6. Under assumptions (H1)-(H4), if there exists a constant $L>0$ such that, for any $t>0$,

$$
\left|\prod_{0 \leq t_{k}<t} J_{k i}\right| \leq L, \quad i=1,2, \ldots, n,
$$

and the zero solution of (2) is p-stable (exponentially p-stable, asymptotically stable), then the zero solution of (6) is also pstable (exponentially p-stable, asymptotically stable).

Combining Theorems 5 and 6 , one can easily obtain the following results.
Theorem 7. Assume that (H1)-(H4) hold and inequalities (11) and (13) are satisfied, then the zero solution of (2) is p-stable (exponentially p-stable, asymptotically stable) if and only if the zero solution of (6) is also p-stable (exponentially p-stable, asymptotically stable).

Remark 8. For impulsive neural networks, many researchers supposed that the impulsive operators are linear (e.g., [7, 12$15])$; that is,

$$
x_{i}\left(t_{k}^{+}\right)-x_{i}\left(t_{k}\right)=-\gamma_{k i} x_{i}\left(t_{k}\right), \quad 0<\gamma_{k i} \leq 2 .
$$

From the definition of $J_{k i}$ in Section 2, we have $\left|J_{k i}^{-1}\right|=\mid 1-$ $\gamma_{k i} \mid<1$; then $\left|\prod_{0 \leq t_{k}<t} J_{k i}^{-1}\right| \leq 1$. Obviously, the condition (11) in Theorem 5 is less conservative than (14), in which (11) ensures that the stability of the delayed stochastic reactiondiffusion differential system without impulses can be used to judge the stability of the corresponding system with impulses.

Remark 9. In $[2,8]$, the authors dealt with the stochastic differential systems with delay and nonlinear impulsive effects, the stability results of which were showed by transforming the system into a corresponding system without impulses. However, the distributed delays and diffusion effects were not taken into account in the previous systems. In this paper, we incorporated stochastic perturbations, reaction-diffusion effects, and mixed time delays into impulsive differential system and derived the stability criteria of the system. It is readily seen that our results are more general than those reported in $[2,8]$.

\section{Application to Impulsive Stochastic Reaction-Diffusion Neural Networks}

In this section, we apply our previous stability results to analyze the stability of the following ISRDCGNNs with time delays:

$$
\begin{aligned}
\mathrm{d} u_{i}(t, x)=\sum_{l=1}^{m} \frac{\partial}{\partial x_{l}}\left(D_{i l}\left(t, x, u_{i}(t, x)\right) \frac{\partial u_{i}(t, x)}{\partial x_{l}}\right) \mathrm{d} t \\
-\alpha_{i}\left(u_{i}(t, x)\right)\left[\beta_{i}\left(u_{i}(t, x)\right)\right. \\
\quad-\sum_{j=1}^{n} a_{i j} f_{j}\left(u_{j}(t, x)\right) \\
-\sum_{j=1}^{n} b_{i j} g_{j}\left(u_{j}\left(t-\tau_{i j}(t), x\right)\right) \\
\left.\quad-\sum_{j=1}^{n} c_{i j} h_{j}\left(\int_{t-r(t)}^{t} u_{j}(s, x) \mathrm{d} s\right)\right] \mathrm{d} t
\end{aligned}
$$




$$
\begin{gathered}
+\sum_{j=1}^{n} \sigma_{i j}\left(t, u_{j}(t, x), u_{j}\left(t-\tau_{i j}(t), x\right),\right. \\
\left.\int_{t-r(t)}^{t} u_{j}(s, x) \mathrm{d} s\right) \mathrm{d} w_{i}(t), \\
t \neq t_{k}, \\
u_{i}\left(t_{k}^{+}, x\right)-u_{i}\left(t_{k}, x\right)=I_{k i}\left(u_{1}\left(t_{k}, x\right), \ldots, u_{n}\left(t_{k}, x\right)\right), \\
t=t_{k}, \quad k \in N, \quad i=1,2, \ldots, n,
\end{gathered}
$$

where $u(t, x)=\left(u_{1}(t, x), u_{2}(t, x), \ldots, u_{n}(t, x)\right)^{\mathrm{T}} \in \mathbb{R}^{n}$ denotes the state vector associated with the neurons, $x \in \Omega \subset \mathbb{R}^{m}$, and $\Omega=\left\{x=\left(x_{1}, x_{2}, \ldots, x_{m}\right)^{\mathrm{T}}|| x_{k} \mid<l_{k}, k=1,2, \ldots, m\right\}$ is a bounded compact set with smooth boundary $\partial \Omega$ and mes $\Omega>0, D_{i l}\left(t, x, u_{i}(t, x)\right) \geq 0$ denotes the diffusion function, and let $D_{i l}=\sup _{t \geq 0, x \in \Omega} D_{i l}\left(t, x, u_{i}(t, x)\right) ;\left(a_{i j}\right)_{n \times n}$, $\left(b_{i j}\right)_{n \times n}$ and $\left(c_{i j}\right)_{n \times n}$ are the interconnection weight matrices, $\alpha_{i}\left(u_{i}(t, x)\right)$ represents an amplification function, $\beta_{i}\left(u_{i}(t, x)\right)$ is an appropriately behaved function, $f_{j}, g_{j}$, and $h_{j}$ denote the activation functions, and $\left(\sigma_{i j}\right)_{n \times n}$ is the diffusion coefficient matrix; $\sigma_{i}=\left(\sigma_{i 1}, \sigma_{i 2}, \ldots, \sigma_{i n}\right), \beta_{i}(0)=f_{i}(0)=g_{i}(0)=h_{i}(0)=$ $\sigma_{i j}(0)=0$, and $\tau_{i j}(t) \leq \tau, r(t) \leq r$. (15) are

The boundary condition and the initial value of system

$$
\begin{aligned}
& \left.u_{i}(t, x)\right|_{\partial \Omega}=0, \quad(t, x) \in[-\delta,+\infty) \times \partial \Omega, i=1,2, \ldots, n, \\
& u_{i}(s, x)=\phi_{i}(s, x), \quad(s, x) \in[-\delta, 0] \times \Omega, i=1,2, \ldots, n,
\end{aligned}
$$

where $\delta=\max \{\tau, r\}$.

Equivalently, we consider the following stochastic reaction-diffusion Cohen-Grossberg neural networks without impulses:

$$
\begin{aligned}
\mathrm{d} v_{i}(t, x) & \sum_{l=1}^{m} \frac{\partial}{\partial x_{l}}\left(D_{i l}\left(t, x, \prod_{0 \leq t_{k}<t} J_{k i}^{-1} v_{i}(t, x)\right) \frac{\partial v_{i}(t, x)}{\partial x_{l}}\right) \mathrm{d} t \\
- & \prod_{0 \leq t_{k}<t} J_{k i} \cdot \alpha_{i}\left(\prod_{0 \leq t_{k}<t} J_{k i}^{-1} v_{i}(t, x)\right) \\
\times & \beta_{i}\left(\prod_{0 \leq t_{k}<t} J_{k i}^{-1} v_{i}(t, x)\right) \\
& -\sum_{j=1}^{n} a_{i j} f_{j}\left(\prod_{0 \leq t_{k}<t} J_{k j}^{-1} v_{j}(t, x)\right) \\
& -\sum_{j=1}^{n} b_{i j} g_{j}\left(\prod_{0 \leq t_{k}<t-\tau_{i j}(t)} J_{k j}^{-1} v_{j}\left(t-\tau_{i j}(t), x\right)\right) \\
& \left.-\sum_{j=1}^{n} c_{i j} h_{j}\left(\int_{t-r(t)}^{t} \prod_{0 \leq t_{k}<s} J_{k s}^{-1} v_{j}(s, x) \mathrm{d} s\right)\right] \mathrm{d} t
\end{aligned}
$$

$$
\begin{array}{rl}
+\prod_{0 \leq t_{k}<t} J_{k i} \cdot \sum_{j=1}^{n} \sigma_{i j}(t, & \prod_{0 \leq t_{k}<t} J_{k j}^{-1} v_{j}(t, x), \\
& \prod_{0 \leq t_{k}<t-\tau_{i j}(t)} J_{k j}^{-1} v_{j}\left(t-\tau_{i j}(t), x\right) \\
& \left.\int_{t-r(t)}^{t} \prod_{0 \leq t_{k}<s} J_{k j}^{-1} v_{j}(s, x) \mathrm{d} s\right) \mathrm{d} w_{i}(t), \\
i & i=1,2, \ldots, n .
\end{array}
$$

Throughout this section, we make the following assumptions:

(H5) $\alpha_{i}(u)$ is a continuous function, and $0<\underline{\alpha}_{i} \leq \alpha_{i}(u) \leq$ $\bar{\alpha}_{i}$ for all $u \in \mathbb{R}, i=1,2, \ldots, n$;

(H6) there exists a constant $\beta_{i}>0$ such that

$$
\frac{\beta_{i}(u)-\beta_{i}(v)}{u-v} \geq \beta_{i}
$$

for all $u, v \in \mathbb{R}(u \neq v), i=1,2, \ldots, n ;$

(H7) there exist positive constants $F_{i}, G_{i}$, and $H_{i}$ such that

$$
\begin{gathered}
F_{i}=\sup _{u \neq v}\left|\frac{f_{i}(u)-f_{i}(v)}{u-v}\right|, \quad G_{i}=\sup _{u \neq v}\left|\frac{g_{i}(u)-g_{i}(v)}{u-v}\right|, \\
H_{i}=\sup _{u \neq v}\left|\frac{h_{i}(u)-h_{i}(v)}{u-v}\right|,
\end{gathered}
$$

for all $u, v \in \mathbb{R}(u \neq v), i=1,2, \ldots, n ;$

(H8) there exist positive constants $s_{i j}^{(1)}, s_{i j}^{(2)}$, and $s_{i j}^{(3)}(i, j=$ $1,2, \ldots, n)$ such that

$$
\begin{aligned}
&\left(\sigma_{i}\left(t, u^{(1)}, u^{(2)}, u^{(3)}\right)-\sigma_{i}\left(t, v^{(1)}, v^{(2)}, v^{(3)}\right)\right) \\
& \quad \times\left(\sigma_{i}\left(t, u^{(1)}, u^{(2)}, u^{(3)}\right)-\sigma_{i}\left(t, v^{(1)}, v^{(2)}, v^{(3)}\right)\right)^{\mathrm{T}} \\
& \leq \sum_{j=1}^{n}\left(s_{i j}^{(1)}\left|u_{j}^{(1)}-v_{j}^{(1)}\right|^{2}\right. \\
&\left.\quad+s_{i j}^{(2)}\left|u_{j}^{(2)}-v_{j}^{(2)}\right|^{2}+s_{i j}^{(3)}\left|u_{j}^{(3)}-v_{j}^{(3)}\right|^{2}\right)
\end{aligned}
$$
results.

for all $u^{(k)}=\left(u_{1}^{(k)}, u_{2}^{(k)}, \ldots, u_{n}^{(k)}\right), v^{(k)}=\left(v_{1}^{(k)}, v_{2}^{(k)}, \ldots\right.$, $\left.v_{n}^{(k)}\right), k=1,2,3$.

The following lemmas are useful in proving our main

Lemma 10 (see [16]). Let $Q$ be an $n \times n$ matrix with nonpositive off-diagonal elements; then $Q$ is an M-matrix if and only if there exists a vector $\xi>0$ such that $Q \xi>0$.

Lemma 11 (see [10]). Let $p \geq 2$ be a positive integer, $l_{k}(k=1,2, \ldots, m)$ positive constants, and cube 
$\Omega=\left\{x=\left(x_{1}, x_{2}, \ldots, x_{m}\right)^{\mathrm{T}} \in \mathbb{R}^{m}|| x_{k} \mid<l_{k}, k=1,2, \ldots, m\right\}$. Let $h(x)$ be a real-valued function belonging to $C^{1}(\Omega)$ which vanishes on the boundary $\partial \Omega$ of $\Omega$, that is, $\left.h(x)\right|_{\partial \Omega=0}$. Then

$$
\begin{array}{r}
\int_{\Omega}|h(x)|^{p} \mathrm{~d} x \leq \frac{p^{2} l_{k}^{2}}{4} \int_{\Omega}|h(x)|^{p-2}\left|\frac{\partial h(x)}{\partial x_{k}}\right|^{2} \mathrm{~d} x, \\
k=1,2, \ldots, m .
\end{array}
$$

Lemma 12 (see [17]). Let $a, b \geq 0, p \geq i>0$; then

$$
a^{p-i} b^{i} \leq \frac{p-i}{p} a^{p}+\frac{i}{p} b^{p}
$$

Theorem 13. Under assumptions (H5)-(H8), if inequalities (11) and (13) are satisfied and $Q+T$ is an M-matrix, where

$$
\begin{gathered}
Q=\left(q_{i j}\right)_{n \times n}, \\
q_{i j}=-L M \bar{\alpha}_{i}\left(\left|a_{i j}\right| F_{j}+\left|b_{i j}\right| G_{j}+r\left|c_{i j}\right| H_{j}\right), \\
T=\left(t_{i j}\right)_{n \times n^{\prime}} \\
t_{i j}=-(p-1) L^{2} M^{2}\left(s_{i j}^{(1)}+s_{i j}^{(2)}+r^{2} s_{i j}^{(3)}\right) \quad(i \neq j), \\
t_{i i}=\frac{4(p-1)}{p} \sum_{l=1}^{m} \frac{D_{i l}}{l_{i}^{2}}+p \underline{\alpha}_{i} \beta_{i} \\
-(p-1) L M \bar{\alpha}_{i} \\
\times \sum_{j=1}^{n}\left[\left|a_{i j}\right| F_{j}+\left|b_{i j}\right| G_{j}+r\left|c_{i j}\right| H_{j}\right. \\
\left.+\frac{(p-2) L M}{2 \bar{\alpha}_{i}}\left(s_{i j}^{(1)}+s_{i j}^{(2)}+r^{2} s_{i j}^{(3)}\right)\right] \\
-(p-1) L^{2} M^{2}\left(s_{i i}^{(1)}+s_{i i}^{(2)}+r^{2} s_{i i}^{(3)}\right),
\end{gathered}
$$

then the zero solution of system (15) is exponentially p-stable.

Proof. Since $Q+T$ is an M-matrix, by Lemma 10, there exists $\xi=\left(\xi_{1}, \xi_{2}, \ldots, \xi_{n}\right)^{\mathrm{T}}$ such that $(Q+T) \xi>0$; that is,

$$
\begin{gathered}
{\left[\frac{4(p-1)}{p} \sum_{l=1}^{m} \frac{D_{i l}}{l_{i}^{2}}+p \underline{\alpha}_{i} \beta_{i}-(p-1) L M \bar{\alpha}_{i}\right.} \\
\times \sum_{j=1}^{n}\left(\left|a_{i j}\right| F_{j}+\left|b_{i j}\right| G_{j}+r\left|c_{i j}\right| H_{j}\right. \\
\left.\left.+\frac{(p-2) L M}{2 \bar{\alpha}_{i}}\left(s_{i j}^{(1)}+s_{i j}^{(2)}+r^{2} s_{i j}^{(3)}\right)\right)\right] \xi_{i}
\end{gathered}
$$

$$
\begin{aligned}
&-L M \bar{\alpha}_{i} \sum_{j=1}^{n}\left[\left|a_{i j}\right| F_{j}+\left|b_{i j}\right| G_{j}+r\left|c_{i j}\right| H_{j}\right. \\
&\left.+\frac{(p-1) L M}{\bar{\alpha}_{i}}\left(s_{i j}^{(1)}+s_{i j}^{(2)}+r^{2} s_{i j}^{(3)}\right)\right] \xi_{j}>0 \\
& i=1,2, \ldots, n .
\end{aligned}
$$

We can choose a sufficiently small constant $\epsilon>0$ such that

$$
\begin{gathered}
{\left[\frac{4(p-1)}{p} \sum_{l=1}^{m} \frac{D_{i l}}{l_{i}^{2}}+p \underline{\alpha}_{i} \beta_{i}-\epsilon-(p-1) L M \bar{\alpha}_{i}\right.} \\
\times \sum_{j=1}^{n}\left(\left|a_{i j}\right| F_{j}+\left|b_{i j}\right| G_{j}+r\left|c_{i j}\right| H_{j}\right. \\
\left.\left.+\frac{(p-2) L M}{2 \bar{\alpha}_{i}}\left(s_{i j}^{(1)}+s_{i j}^{(2)}+r^{2} s_{i j}^{(3)}\right)\right)\right] \xi_{i} \\
-L M \bar{\alpha}_{i} \sum_{j=1}^{n}\left[\left(\left|a_{i j}\right| F_{j}+\frac{(p-1) L M}{\bar{\alpha}_{i}} s_{i j}^{(1)}\right)\right. \\
+e^{\epsilon \tau}\left(\left|b_{i j}\right| G_{j}+\frac{(p-1) L M}{\bar{\alpha}_{i}} s_{i j}^{(2)}\right) \\
\left.+\frac{e^{\epsilon r-1}}{\epsilon}\left(\left|c_{i j}\right| H_{j}+\frac{(p-1) L M r}{\bar{\alpha}_{i}} s_{i j}^{(3)}\right)\right] \xi_{j}>0 .
\end{gathered}
$$

Let $w_{i}(t, x)=e^{\epsilon t} \int_{\Omega}\left|v_{i}(t, x)\right|^{p} \mathrm{~d} x, p \geq 2, i=1,2, \ldots, n$. By the Itô differential formula, the stochastic derivative of $w_{i}(t, x)$ along (17) can be derived as follows:

$$
\begin{aligned}
& L w_{i}(t, x) \\
& =\epsilon e^{\epsilon t} \int_{\Omega}\left|v_{i}(t, x)\right|^{p} \mathrm{~d} x \\
& +p e^{\epsilon t} \operatorname{sgn}\left(v_{i}(t, x)\right) \int_{\Omega}\left|v_{i}(t, x)\right|^{p-1} \\
& \times\left[\sum_{l=1}^{m} \frac{\partial}{\partial x_{l}}\left(D_{i l}\left(t, x, \prod_{0 \leq t_{k}<t} J_{k i}^{-1} v_{i}(t, x)\right) \frac{\partial v_{i}(t, x)}{\partial x_{l}}\right)\right. \\
& -\prod_{0 \leq t_{k}<t} J_{k i} \cdot \alpha_{i}\left(\prod_{0 \leq t_{k}<t} J_{k i}^{-1} v_{i}(t, x)\right) \\
& \times\left[\beta_{i}\left(\prod_{0 \leq t_{k}<t} J_{k i}^{-1} v_{i}(t, x)\right)\right. \\
& -\sum_{j=1}^{n} a_{i j} f_{j}\left(\prod_{0 \leq t_{k}<t} J_{k j}^{-1} v_{j}(t, x)\right) \\
& -\sum_{j=1}^{n} b_{i j} g_{j}\left(\prod_{0 \leq t_{k}<t-\tau_{i j}(t)} J_{k j}^{-1} v_{j}\left(t-\tau_{i j}(t), x\right)\right)
\end{aligned}
$$




$$
\begin{array}{r}
\left.-\sum_{j=1}^{n} c_{i j} h_{j}\left(\int_{t-r(t)}^{t} \prod_{0 \leq t_{k}<s} J_{k s}^{-1} v_{j}(s, x) \mathrm{d} s\right)\right] \mathrm{d} x \\
+\frac{p(p-1)}{2} e^{\epsilon t}\left(\prod_{0 \leq t_{k}<t} J_{k i}\right)^{2} \int_{\Omega}\left|v_{i}(t, x)\right|^{p-2} \sigma_{i} \sigma_{i}^{\mathrm{T}} \mathrm{d} x \\
i=1,2, \ldots, n .
\end{array}
$$

By Lemma 11, we derive from (16) that

$$
\begin{aligned}
& p \operatorname{sgn}(\left.v_{i}(t, x)\right) \int_{\Omega}\left|v_{i}(t, x)\right|^{p-1} \\
& \times \sum_{l=1}^{m} \frac{\partial}{\partial x_{l}}\left(D_{i l}\left(t, x, \prod_{0 \leq t_{k}<t} J_{k i}^{-1} v_{i}(t, x)\right)\right. \\
&\left.\times \frac{\partial v_{i}(t, x)}{\partial x_{l}}\right) \mathrm{d} x \\
&=p \int_{\Omega}\left|v_{i}(t, x)\right|^{p-2} v_{i}(t, x) \nabla \\
& \quad\left(D_{i l}\left(t, x, \prod_{0 \leq t_{k}<t} J_{k i}^{-1} v_{i}(t, x)\right) \frac{\partial v_{i}(t, x)}{\partial x_{l}}\right)_{l=1}^{m} \mathrm{~d} x \\
& \leq-p(p-1) \int_{\Omega} \sum_{l=1}^{m} D_{i l}\left|v_{i}(t, x)\right|^{p-2}\left(\frac{\partial v_{i}(t, x)}{\partial x_{l}}\right)^{2} \mathrm{~d} x \\
& \leq-\frac{4(p-1)}{p} \sum_{l=1}^{m} \frac{D_{i l}}{l_{i}^{2}} \int_{\Omega}\left|v_{i}(t, x)\right|^{p} \mathrm{~d} x .
\end{aligned}
$$

Applying assumptions (H5)-(H8) and Lemma 12, we can deduce from (11), (13), and (27) that

$$
\begin{aligned}
L w_{i}(t, x) \leq e^{\epsilon t} \int_{\Omega}[( & \left.-\frac{4(p-1)}{p} \sum_{l=1}^{m} \frac{D_{i l}}{l_{i}^{2}}-p \underline{\alpha}_{i} \beta_{i}\right) \\
& \times\left|v_{i}(t, x)\right|^{p} \\
& +p L M \bar{\alpha}_{i}\left|v_{i}(t, x)\right|^{p-1} \\
& \times \sum_{j=1}^{n}\left(\left|a_{i j}\right| F_{j}\left|v_{j}(t, x)\right|\right. \\
& +\left|b_{i j}\right| G_{j}\left|v_{j}\left(t-\tau_{i j}(t), x\right)\right| \\
& \left.+\frac{p(p-1)}{2} L^{2} M^{2}\left|v_{i}(t, x)\right|_{t-r(t)}^{t-2}\left|v_{j}(s, x)\right| \mathrm{d} s\right)
\end{aligned}
$$

$$
\begin{aligned}
& \times \sum_{j=1}^{n}\left(s_{i j}^{(1)} v_{j}^{2}(t, x)\right. \\
& \quad+s_{i j}^{(2)} v_{j}^{2}\left(t-\tau_{i j}(t), x\right) \\
& \left.\left.\quad+r s_{i j}^{(3)} \int_{t-r(t)}^{t} v_{j}^{2}(s, x) \mathrm{d} s\right)\right] \mathrm{d} x
\end{aligned}
$$$$
\leq e^{\epsilon t} \int_{\Omega}\left[\left(\epsilon-\frac{4(p-1)}{p} \sum_{l=1}^{m} \frac{D_{i l}}{l_{i}^{2}}-p \underline{\alpha}_{i} \beta_{i}\right)\right.
$$

$$
\times\left|v_{i}(t, x)\right|^{p}
$$$$
+(p-1) L M \bar{\alpha}_{i}\left|v_{i}(t, x)\right|^{p}
$$$$
\times \sum_{j=1}^{n}\left(\left|a_{i j}\right| F_{j}+\left|b_{i j}\right| G_{j}+r\left|c_{i j}\right| H_{j}\right)
$$$$
+L M \bar{\alpha}_{i} \sum_{j=1}^{n}\left(\left|a_{i j}\right| F_{j}\left|v_{j}(t, x)\right|^{p}+\left|b_{i j}\right|\right.
$$$$
\times G_{j}\left|v_{j}\left(t-\tau_{i j}(t), x\right)\right|^{p}
$$$$
+\left|c_{i j}\right| H_{j}
$$$$
\left.\times \int_{t-r(t)}^{t}\left|v_{j}(s, x)\right|^{p} \mathrm{~d} s\right)
$$$$
+\frac{(p-1)(p-2)}{2} L^{2} M^{2}\left|v_{i}(t, x)\right|^{p}
$$$$
\times \sum_{j=1}^{n}\left(s_{i j}^{(1)}+s_{i j}^{(2)}+r^{2} s_{i j}^{(3)}\right)
$$$$
+(p-1) L^{2} M^{2}
$$$$
\times \sum_{j=1}^{n}\left(s_{i j}^{(1)}\left|v_{j}(t, x)\right|^{p}\right.
$$$$
+s_{i j}^{(2)}\left|v_{j}\left(t-\tau_{i j}(t), x\right)\right|^{p}
$$$$
+r s_{i j}^{(3)}
$$$$
\left.\left.\times \int_{t-r(t)}^{t}\left|v_{j}(s, x)\right|^{p}\right) \mathrm{~d} s\right] \mathrm{d} x
$$

$$
\leq\left[\epsilon-\frac{4(p-1)}{p} \sum_{l=1}^{m} \frac{D_{i l}}{l_{i}^{2}}-p \underline{\alpha}_{i} \beta_{i}+(p-1) L M \bar{\alpha}_{i}\right.
$$$$
\times \sum_{j=1}^{n}\left(\left|a_{i j}\right| F_{j}+\left|b_{i j}\right| G_{j}+r\left|c_{i j}\right| H_{j}\right.
$$$$
+\frac{(p-2) L M}{2 \bar{\alpha}_{i}}
$$$$
\left.\left.\times\left(s_{i j}^{(1)}+s_{i j}^{(2)}+r^{2} s_{i j}^{(3)}\right)\right)\right] w_{i}(t, x)
$$ 


$$
\begin{aligned}
+L M \bar{\alpha}_{i} \sum_{j=1}^{n}[ & \left(\left|a_{i j}\right| F_{j}+\frac{(p-1) L M}{\bar{\alpha}_{i}} s_{i j}^{(1)}\right) \\
& \times w_{j}(t, x) \\
& +e^{\epsilon \tau}\left(\left|b_{i j}\right| G_{j}\right. \\
& \left.\times \frac{(p-1) L M}{\bar{\alpha}_{i}} s_{i j}^{(2)}\right) \\
& +\left(\mid w_{i j}\left(t-\tau_{i j}(t), x\right)\right. \\
& \left.\times \int_{t-r(t)}^{t} e^{\epsilon(t-s)} w_{j}(s, x) \mathrm{d} s\right] .
\end{aligned}
$$

Further, we can get

$$
\begin{gathered}
D^{+}\left(E w_{i}(t, x)\right) \\
\leq\left[\epsilon-\frac{4(p-1)}{p} \sum_{l=1}^{m} \frac{D_{i l}}{l_{i}^{2}}\right. \\
\quad-p \underline{\alpha}_{i} \beta_{i}+(p-1) L M \bar{\alpha}_{i} \\
\quad+\sum_{j=1}^{n}\left(\left|a_{i j}\right| F_{j}+\left|b_{i j}\right| G_{j}+r\left|c_{i j}\right| H_{j}\right. \\
\left.\left.+\frac{(p-2) L M}{2 \bar{\alpha}_{i}}\left(s_{i j}^{(1)}+s_{i j}^{(2)}+r^{2} s_{i j}^{(3)}\right)\right)\right] E w_{i}(t, x) \\
+L \bar{\alpha}_{i} \sum_{j=1}^{n}\left[\left(\left|a_{i j}\right| F_{j}+\frac{(p-1) L M}{\bar{\alpha}_{i}} s_{i j}^{(1)}\right) E w_{j}(t, x)\right. \\
+e^{\epsilon \tau}\left(\left|b_{i j}\right| G_{j}+\frac{(p-1) L M}{\bar{\alpha}_{i}} s_{i j}^{(2)}\right) \\
\left.\times \int_{t-r(t)}^{t} e^{\epsilon(t-s)} E w_{j}(s, x) \mathrm{d} s\right] . \\
+\left(\left|c_{i j}\right| H_{j}+\frac{(p-1) L M r}{\bar{\alpha}_{i}} s_{i j}^{(3)}\right)
\end{gathered}
$$

Denoting $k_{0}=\|\phi\|^{p} / \min _{1 \leq i \leq n}\left\{\xi_{i}\right\}$, we have

$$
\begin{aligned}
E w_{i}(t, x) & =e^{\epsilon t} E\left\|v_{i}(t, x)\right\|^{p} \leq E\left\|v_{i}(t, x)\right\|^{p} \\
& \leq k_{0} \xi_{i}, \quad t \in[-\delta, 0], i=1,2, \ldots, n .
\end{aligned}
$$

In what follows, we prove that

$$
E w_{i}(t, x) \leq k_{0} \xi_{i}, \quad t \geq 0, i=1,2, \ldots, n .
$$

In fact, if (31) is not true, then there exist $i_{0} \in\{1,2, \ldots, n\}$ and $t^{*} \in[0,+\infty)$ such that

$$
\begin{gathered}
E w_{i_{0}}\left(t^{*}, x\right) \leq k_{0} \xi_{i_{0}}, \quad D^{+} E w_{i_{0}}\left(t^{*}, x\right)>0, \\
E w_{j}(t, x) \leq k_{0} \xi_{j}, \quad t \in\left[-\delta, t^{*}\right], \quad j=1,2, \ldots, n .
\end{gathered}
$$

However, (25), (29), and (32) imply that

$$
\begin{gathered}
D^{+}\left(E w_{i_{0}}\left(t^{*}, x\right)\right) \\
\leq k_{0}\left\{\left[\epsilon-\frac{4(p-1)}{p} \sum_{l=1}^{m} \frac{D_{i_{0} l}}{l_{i_{0}}^{2}}-p \underline{\alpha}_{i_{0}} \beta_{i_{0}}+(p-1) L M \bar{\alpha}_{i_{0}}\right.\right. \\
\quad \times \sum_{j=1}^{n}\left(\left|a_{i_{0} j}\right| F_{j}\right. \\
+\left|b_{i_{0} j}\right| G_{j}+r\left|c_{i_{0} j}\right| H_{j} \\
\left.\left.+\frac{(p-2) L M}{2 \bar{\alpha}_{i_{0}}}\left(s_{i_{0} j}^{(1)}+s_{i_{0} j}^{(2)}+r^{2} s_{i_{0} j}^{(3)}\right)\right)\right] \xi_{i_{0}} \\
+L M \bar{\alpha}_{i_{0}}\left[\left(\left|a_{i_{0} j}\right| F_{j}+\frac{(p-1) L M}{\bar{\alpha}_{i_{0}}} s_{i_{0} j}^{(1)}\right)\right. \\
\left.\left.\left.\quad \sum_{j=1}^{n}+\frac{(p-1) L M r}{\bar{\alpha}_{i_{0}}} s_{i_{0} j}^{(3)}\right)\right] \xi_{j}\right\} \\
+e^{\epsilon \tau}\left(\left|b_{i_{0} j}\right| G_{j}+\frac{(p-1) L M}{\bar{\alpha}_{i_{0}}} s_{i_{0} j}^{(2)}\right) \\
+\frac{e^{\epsilon r}-1}{\epsilon}\left(\left|c_{i_{0} j}\right| H_{j}\right.
\end{gathered}
$$$$
<0,
$$

which is a contradiction. Hence, (31) holds, which leads to

$$
E\left\|v_{i}(t, x)\right\|^{p} \leq k_{0} \xi_{i} e^{-\epsilon t}, \quad t \geq 0, i=1,2, \ldots, n .
$$

Therefore,

$$
E\|v(t, x)\|^{p} \leq K\|\phi\|^{p} e^{-\epsilon t}, \quad t \geq 0,
$$

where $K=\left(\max _{1 \leq i \leq n}\left\{\xi_{i}\right\} / \min _{1 \leq i \leq n}\left\{\xi_{i}\right\}\right) n^{p}$. This means that the zero solution of system (17) is exponentially $p$-stable and the exponential convergence rate equals $\epsilon$. By Theorem 5, we can obtain that the zero solution of system (15) is exponentially $p$-stable. The proof is complete.

Remark 14. The stability of impulsive Cohen-Grossberg neural networks without spacial diffusion or distributed delays or stochastic disturbance, which are special cases of system (15), have been studied in $[5,13,18-20]$. It should be noted that the main result in [13] is a special case of Theorem 13. Further, the stability criteria derived in $[5,18-20]$ are dependent on the 
intervals of adjoining impulsive moments, while our results are independent of that. Thus, our results are new, and they effectually complement or improve the previously published results.

Remark 15. In $[19,21-26]$, the reaction-diffusion neural networks have been investigated. Nevertheless, the diffusion terms were eliminated by inequality analysis techniques, and the derived conditions for the stability of neural networks are the same as those obtained in the cases when there are no reaction-diffusion terms in the systems. Thus, our results including reaction-diffusion terms are less conservative than those in [19, 21-26].

Remark 16. As far as we know, almost all the existing results concerning the stability of neural networks are based on 2norm (e.g., $[5,11,19,21,22,26-28])$. In this paper, we derived the stability criteria of ISRDCGNNs with mixed time delays in terms of $p$-norm. Hence, our results generalize and improve the existing results reported in the previous literature.

\section{Numerical Example}

In this section, we give an example to illustrate the main theoretical results in Sections 3 and 4.

In system (15), let

$$
\begin{aligned}
& n=m=2, \quad \alpha_{1}(x)=2.5+0.5 \cos x, \\
& \alpha_{2}(x)=3-\sin x, \quad \beta_{1}(x)=8 x, \\
& \beta_{2}(x)=10 x, \\
& f_{j}(x)=g_{j}(x)=h_{j}(x) \\
& =\frac{1}{2}(|x+1|+|x-1|), \quad l_{j}=1 \quad(j=1,2), \\
& r(t)=1+\sin t, \quad\left(D_{i l}(x)\right)=\left(\begin{array}{ll}
6 & 6 \\
8 & 8
\end{array}\right), \\
& \left(a_{i j}\right)=\left(\begin{array}{cc}
0.1 & -0.2 \\
-0.3 & -0.5
\end{array}\right), \quad\left(b_{i j}\right)=\left(\begin{array}{cc}
0.8 & 0.8 \\
0.4 & 0.2
\end{array}\right) \text {, } \\
& \left(c_{i j}\right)=\left(\begin{array}{ll}
0.3 & -0.2 \\
0.1 & -0.2
\end{array}\right) \text {, } \\
& \left(\tau_{i j}(t)\right)=\left(\begin{array}{cc}
0.02 \sin ^{2}(t) & 0.01|\cos t| \\
0.03 \cos ^{2}(t) & 0.02|\sin 2 t|
\end{array}\right), \\
& \left(\sigma_{i j}(x, y, z)\right) \\
& =\left(\begin{array}{cc}
0.1 x-0.2 y-0.1 z & 0.2 x+0.3 y+0.1 z \\
0.5 x+0.4 y-0.3 z & 0.3 x+0.1 y+0.2 z
\end{array}\right), \\
& I_{k 1}\left(u_{1}\left(t_{k}, x\right), u_{2}\left(t_{k}, x\right)\right) \\
& =\frac{1}{2^{k+2}} u_{1}\left(t_{k}, x\right) \\
& +\frac{1}{(2 k)^{2}} u_{1}\left(t_{k}, x\right) \sin ^{2}\left(u_{2}\left(t_{k}, x\right)\right),
\end{aligned}
$$

$$
\begin{aligned}
I_{k 2}\left(u_{1}\left(t_{k}, x\right), u_{2}\left(t_{k}, x\right)\right) \\
=\frac{1}{2^{2} 3^{k}} u_{2}\left(t_{k}, x\right) \\
\quad+\frac{1}{(2 k)^{2}} u_{2}\left(t_{k}, x\right) \cos ^{2}\left(u_{1}\left(t_{k}, x\right)\right) .
\end{aligned}
$$

By direct calculation, we obtain that

$$
\begin{gathered}
\underline{\alpha}_{1}=2, \quad \bar{\alpha}_{1}=3, \quad \underline{\alpha}_{2}=2, \\
\bar{\alpha}_{2}=4, \quad \beta_{1}=8, \quad \beta_{2}=10, \\
F_{i}=G_{i}=H_{i}=1, \quad \tau=0.03, \quad r=2, \\
\left(s_{i j}^{(1)}\right)=\left(\begin{array}{cc}
0.04 & 0.12 \\
0.6 & 0.18
\end{array}\right), \quad\left(s_{i j}^{(2)}\right)=\left(\begin{array}{ll}
0.08 & 0.18 \\
0.48 & 0.06
\end{array}\right), \\
\left(s_{i j}^{(3)}\right)=\left(\begin{array}{ll}
0.04 & 0.06 \\
0.36 & 0.12
\end{array}\right), \\
J_{k 1}^{-1}=1+\frac{1}{2^{k+2}}+\frac{1}{(2 k)^{2}} \sin ^{2}\left(u_{2}\left(t_{k}, x\right)\right), \\
J_{k 2}^{-1}=1+\frac{1}{2^{2} 3^{k}}+\frac{1}{(2 k)^{2}} \cos ^{2}\left(u_{1}\left(t_{k}, x\right)\right), \\
L=1, \quad M=2 .
\end{gathered}
$$

Hence, assumptions (H5)-(H8) and inequalities (11) and (13) are satisfied. Taking $p=4$, it is not difficult to compute that

$$
\begin{gathered}
Q=\left(\begin{array}{ll}
-9.0 & -8.4 \\
-7.2 & -8.8
\end{array}\right), \quad T=\left(\begin{array}{cc}
34.60 & -6.48 \\
-30.24 & 32.48
\end{array}\right), \\
Q+T=\left(\begin{array}{cc}
25.60 & -14.88 \\
-37.44 & 23.68
\end{array}\right),
\end{gathered}
$$

in which $Q+T$ is an M-matrix, and all the conditions of Theorem 13 are satisfied. From Theorem 13, we know that the zero solution of system (15) with the parameters and functions above is exponentially 4 -stable (see Figure 1).

\section{Concluding Remark}

In this paper, we incorporated stochastic perturbations, reaction-diffusion effects, and mixed time delays into impulsive differential systems. First, an equivalent relation between the solution of a stochastic reaction-diffusion differential system with time delays and impulsive effects and that of corresponding system without impulses was established. Second, some stability criteria for the stochastic reactiondiffusion differential system with time delays and impulsive effects were derived by transforming the solutions of the system to those of corresponding one without impulses. Third, the stability criteria were applied to ISRDCGNNs with mixed time delays, and sufficient conditions were obtained for the exponential $p$-stability of the zero solution to the neural networks. Lastly, a numerical example was provided to illustrate the effectiveness of our theoretical results. Our 


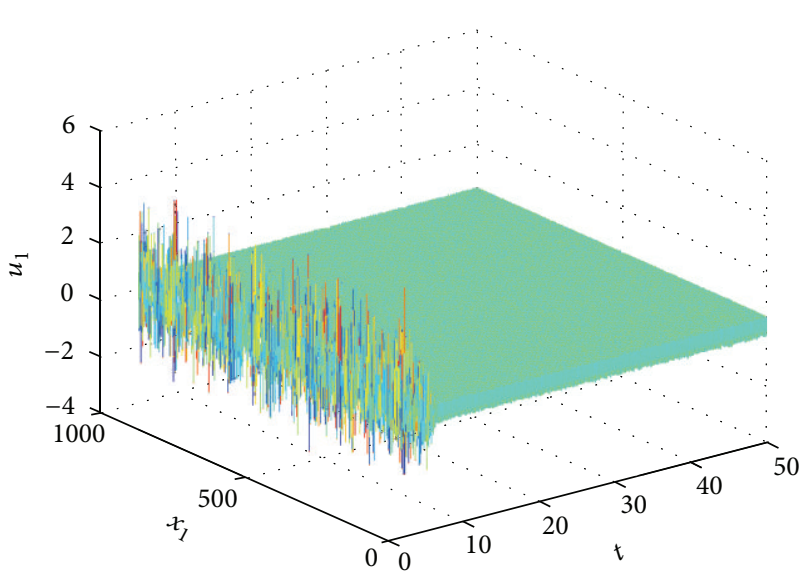

(a)

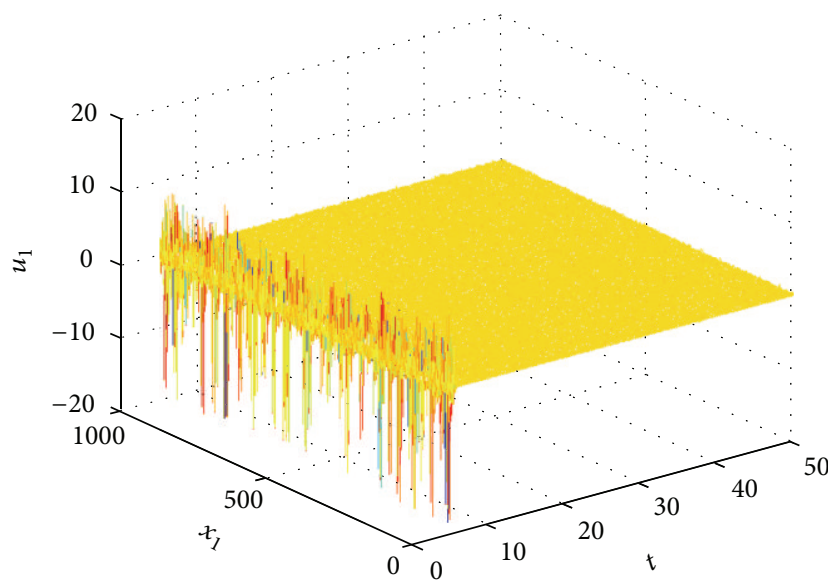

(b)

FIGURE 1: Transient behaviors of the state variables $u_{1}(t, x)$ and $u_{2}(t, x)$ in the example.

stability results provide a new, convenient, and efficient approach to study the stability of stochastic reaction-diffusion differential systems with time delays and impulsive effects, and some previously published results are generalized and improved.

\section{Acknowledgments}

This work was supported by the National Natural Science Foundation of China (11071254, 11371368) and the Natural Science Foundation of Hebei Province (A2013506012).

\section{References}

[1] J. O. Alzabut and T. Abdeljawad, "On existence of a globally attractive periodic solution of impulsive delay logarithmic population model," Applied Mathematics and Computation, vol. 198, no. 1, pp. 463-469, 2008.

[2] C. Li, J. Sun, and R. Sun, "Stability analysis of a class of stochastic differential delay equations with nonlinear impulsive effects," Journal of the Franklin Institute, vol. 347, no. 7, pp. 1186-1198, 2010.

[3] X. Li and X. Fu, "Synchronization of chaotic delayed neural networks with impulsive and stochastic perturbations," Communications in Nonlinear Science and Numerical Simulation, vol. 16, no. 2, pp. 885-894, 2011.

[4] L. Pan and J. Cao, "Exponential stability of impulsive stochastic functional differential equations," Journal of Mathematical Analysis and Applications, vol. 382, no. 2, pp. 672-685, 2011.

[5] J. Pan, X. Liu, and S. Zhong, "Stability criteria for impulsive reaction-diffusion Cohen-Grossberg neural networks with time-varying delays," Mathematical and Computer Modelling, vol. 51, no. 9-10, pp. 1037-1050, 2010.

[6] Q. J. Wu, J. Zhou, and L. Xiang, "Global exponential stability of impulsive differential equations with any time delays," Applied Mathematics Letters, vol. 23, no. 2, pp. 143-147, 2010.

[7] X. Fu and X. Li, "LMI conditions for stability of impulsive stochastic Cohen-Grossberg neural networks with mixed delays," Communications in Nonlinear Science and Numerical Simulation, vol. 16, no. 1, pp. 435-454, 2011.
[8] C. Li, J. Shi, and J. Sun, "Stability of impulsive stochastic differential delay systems and its application to impulsive stochastic neural networks," Nonlinear Analysis: Theory, Methods \& Applications, vol. 74, no. 10, pp. 3099-3111, 2011.

[9] Q. Gan, "Exponential synchronization of stochastic CohenGrossberg neural networks with mixed time-varying and reaction-diffusion via periodially intermittent control," Neural Networks, vol. 31, no. 1, pp. 12-21, 2012.

[10] C. Hu, H. Jiang, and Z. Teng, "Impulsive control and synchronization for delayed neural networks with reaction-diffusion terms," IEEE Transactions on Neural Networks, vol. 21, no. 1, pp. 67-81, 2010.

[11] X. Xu, J. Zhang, and W. Zhang, "Mean square exponential stability of stochastic neural networks with reaction-diffusion terms and delays," Applied Mathematics Letters, vol. 24, no. 1, pp. 5-11, 2011.

[12] J. Pan and S. Zhong, "Dynamical behaviors of impulsive reaction-diffusion Cohen-Grossberg neural network with delays," Neurocomputing, vol. 73, no. 7-9, pp. 1344-1351, 2010.

[13] Q. Song and Z. Wang, "Stability analysis of impulsive stochastic Cohen-Grossberg neural networks with mixed time delays," Physica A, vol. 387, no. 13, pp. 3314-3326, 2008.

[14] I. M. Stamova, R. Ilarionov, and R. Vaneva, "Impulsive control for a class of neural networks with bounded and unbounded delays," Applied Mathematics and Computation, vol. 216, no. 1, pp. 285-290, 2010.

[15] Y. Zhang and Q. G. Wang, "Stationary oscillation for highorder Hopfield neural networks with time delays and impulses," Journal of Computational and Applied Mathematics, vol. 231, no. 1, pp. 473-477, 2009.

[16] Q. Song and J. Cao, "Stability analysis of Cohen-Grossberg neural network with both time-varying and continuously distributed delays," Journal of Computational and Applied Mathematics, vol. 197, no. 1, pp. 188-203, 2006.

[17] J. Cao and J. Liang, "Boundedness and stability for CohenGrossberg neural network with time-varying delays," Journal of Mathematical Analysis and Applications, vol. 296, no. 2, pp. 665685, 2004.

[18] K. Li, "Stability analysis for impulsive Cohen-Grossberg neural networks with time-varying delays and distributed delays," 
Nonlinear Analysis: Real World Applications, vol. 10, no. 5, pp. 2784-2798, 2009.

[19] Z. Li and K. Li, "Stability analysis of impulsive Cohen-Grossberg neural networks with distributed delays and reaction-diffusion terms," Applied Mathematical Modelling, vol. 33, no. 3, pp. 13371348, 2009.

[20] X. Zhang, S. Wu, and K. Li, "Delay-dependent exponential stability for impulsive Cohen-Grossberg neural networks with time-varying delays and reaction-diffusion terms," Communications in Nonlinear Science and Numerical Simulation, vol. 16, no. 3, pp. 1524-1532, 2011.

[21] C. Huang, X. Yang, Y. He, and L. Huang, "Stability of stochastic reaction-diffusion recurrent neural networks with unbounded distributed delays," Discrete Dynamics in Nature and Society, vol. 2011, Article ID 570295, 16 pages, 2011.

[22] Z. Li and R. Xu, "Global asymptotic stability of stochastic reaction-diffusion neural networks with time delays in the leakage terms," Communications in Nonlinear Science and Numerical Simulation, vol. 17, no. 4, pp. 1681-1689, 2012.

[23] X. Lou, B. Cui, and W. Wu, "On global exponential stability and existence of periodic solutions for BAM neural networks with distributed delays and reaction-diffusion terms," Chaos, Solitons \& Fractals, vol. 36, no. 4, pp. 1044-1054, 2008.

[24] Q. Song and Z. Wang, "Dynamical behaviors of fuzzy reactiondiffusion periodic cellular neural networks with variable coefficients and delays," Applied Mathematical Modelling, vol. 33, no. 9, pp. 3533-3545, 2009.

[25] Z. Yang and D. Xu, "Global dynamics for non-autonomous reaction-diffusion neural networks with time-varying delays," Theoretical Computer Science, vol. 403, no. 1, pp. 3-10, 2008.

[26] P. Balasubramaniam and C. Vidhya, "Global asymptotic stability of stochastic BAM neural networks with distributed delays and reaction-diffusion terms," Journal of Computational and Applied Mathematics, vol. 234, no. 12, pp. 3458-3466, 2010.

[27] C. Wang, Y. Kao, and G. Yang, "Exponential stability of impulsive stochastic fuzzy reaction-diffusion Cohen-Grossberg neural networks with mixed delays," Neurocomputing, vol. 89, no. 1, pp. 55-63, 2012.

[28] T. Lv and P. Yan, "Dynamical behaviors of reaction-diffusion fuzzy neural networks with mixed delays and general boundary conditions," Communications in Nonlinear Science and Numerical Simulation, vol. 16, no. 2, pp. 993-1001, 2011. 


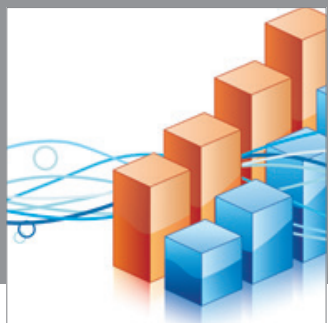

Advances in

Operations Research

mansans

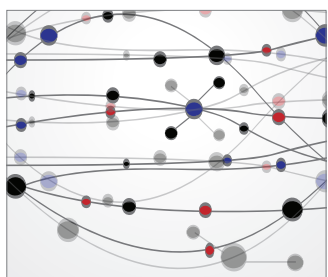

The Scientific World Journal
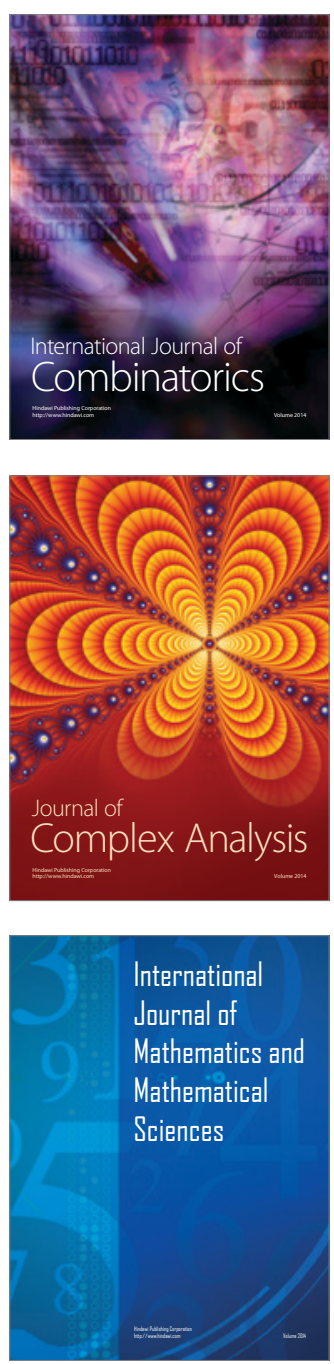
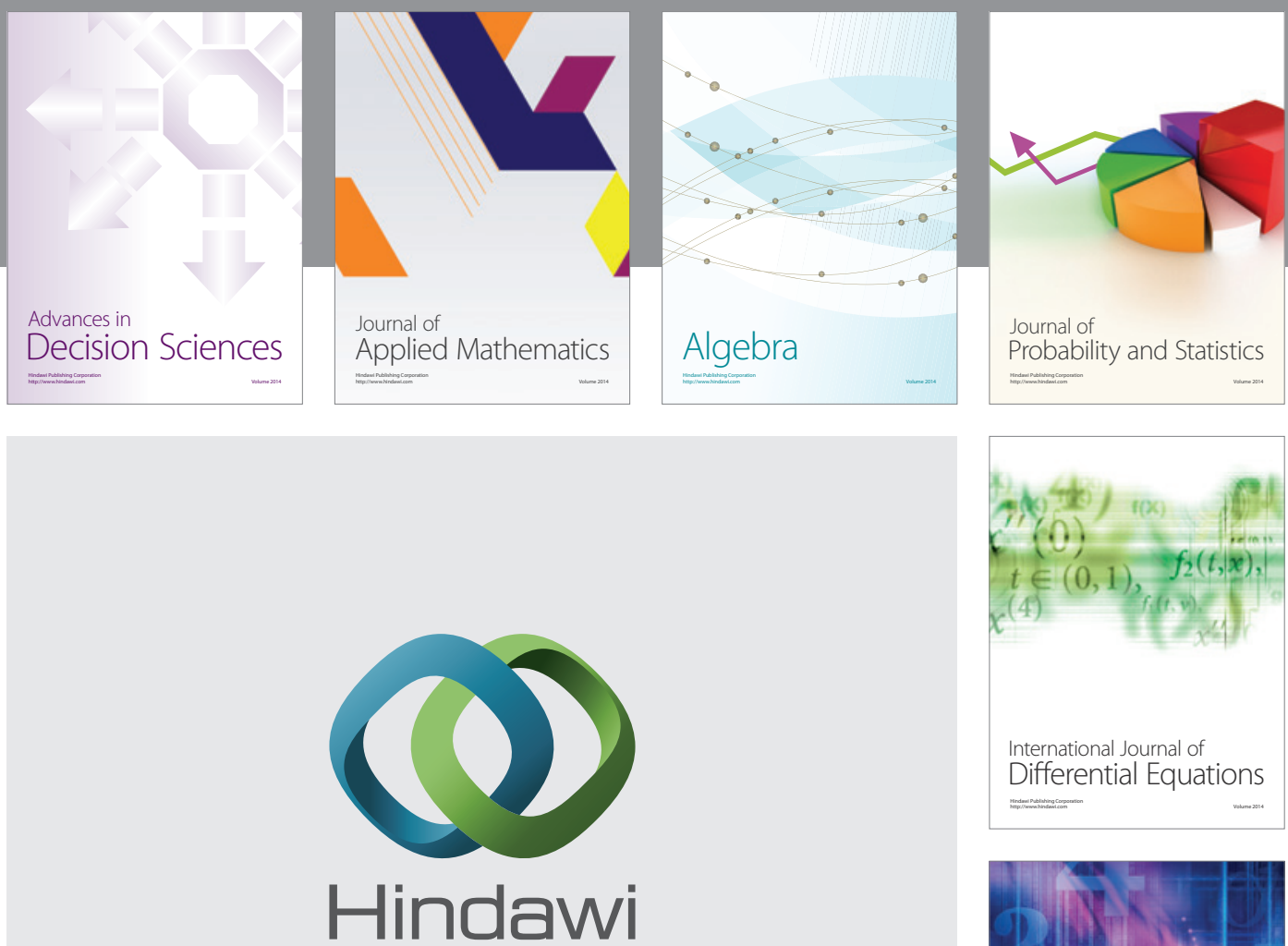

Submit your manuscripts at http://www.hindawi.com
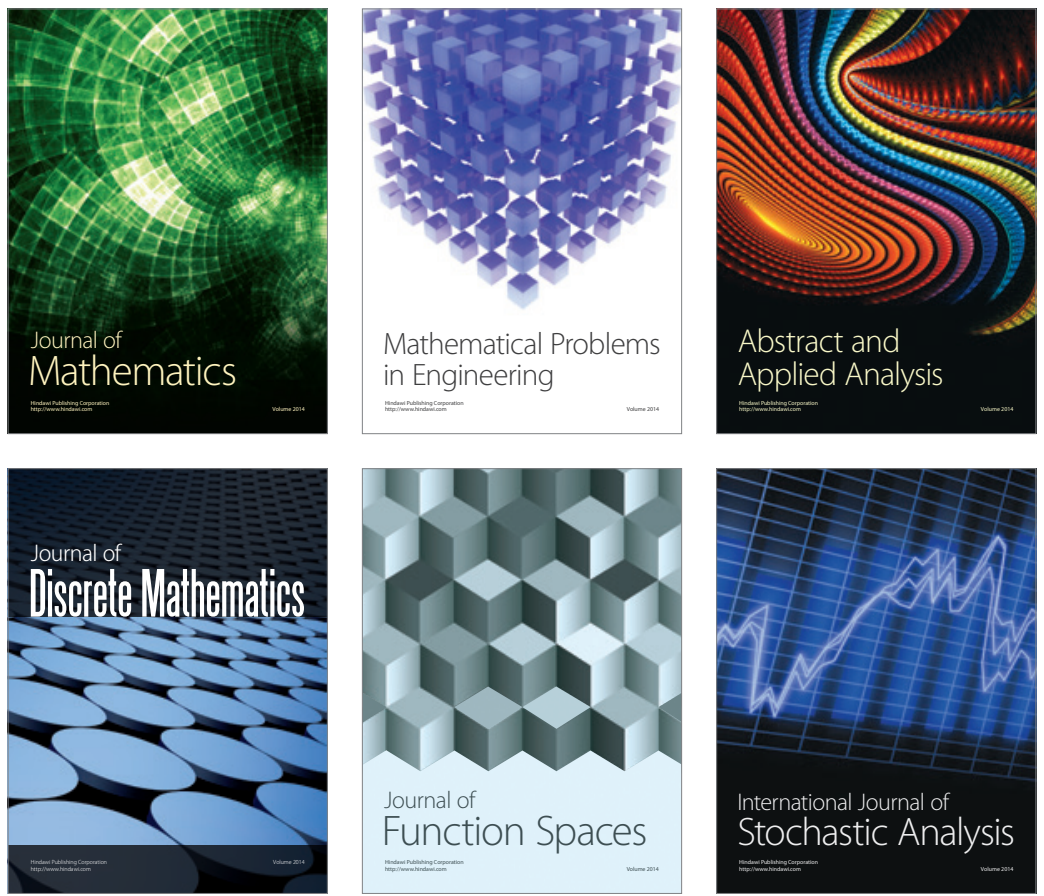

Journal of

Function Spaces

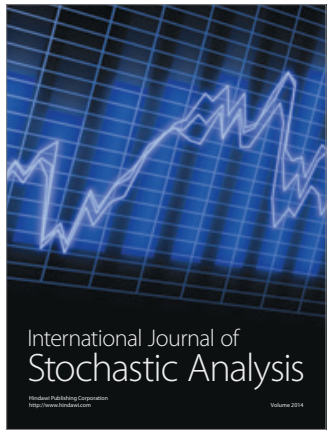

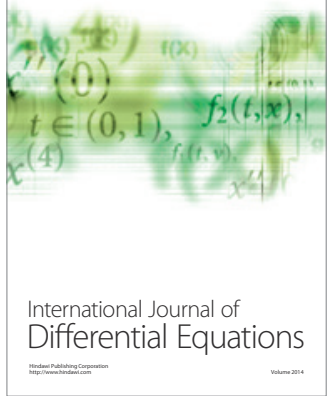
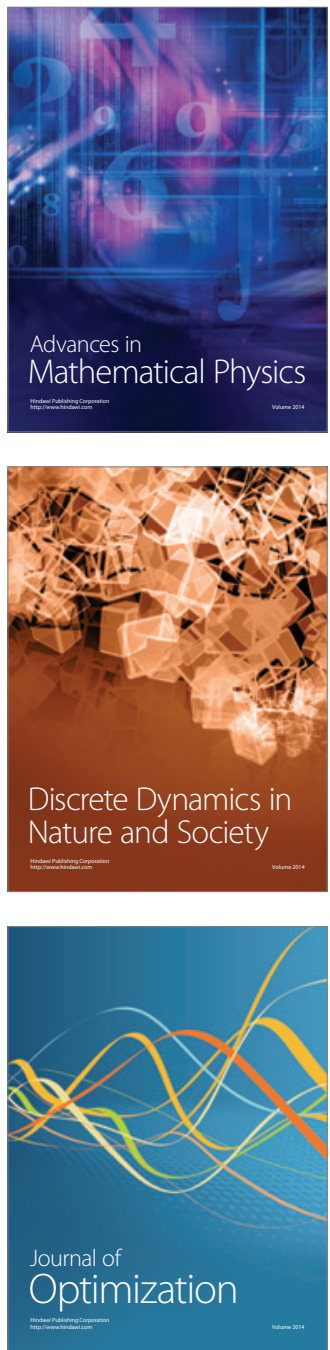